\title{
Reversed tibial locking plates in management of traumatic ipsilateral periprosthetic fractures of the distal femur and proximal tibia after total knee arthroplasty
}

\author{
Rishya Manikam, CS Kumar, Nasir Mohamad
}

\begin{abstract}
Introduction: Ipsilateral distal femoral and proximal tibial periprosthetic fracture following total knee arthroplasty is a rare complication presented to the Emergency Department. Case Report: We report a case of a 5o-year-old man who underwent a right total knee arthroplasty one year ago and presented with periprosthetic fractures following a motor vehicle accident. He sustained comminuted fracture of lower third of right femur and proximal tibia, thus producing "Floating Total Knee" but the knee implants remained intact. He was subsequently treated with two reversed tibial locking plates for the femur and a single locking plate for the tibia. Conclusion: Early and prompt management of this
\end{abstract}

${ }^{1}$ Rishya Manikam, ${ }^{2} \mathrm{CS}$ Kumar, ${ }^{3}$ Nasir Mohamad Affiliations: ${ }^{1}$ Senior Lecturer/ Emergency Consultant, Accident \& Emergency Department, Department of Accident and Emergency Medicine, University Malaya Medical Centre, 59100 Kuala Lumpur, Malaysia; ${ }^{2}$ Senior Lecturer/ Orthopedic Surgeon, Department of Orthopaedic Surgery, University Malaya Medical Centre, 59100 Kuala Lumpur, Malaysia; ${ }^{3}$ Senior Lecturer/ Emergency Consultant, Department of Emergency Medicine, School of Medical Sciences, Health Campus, Universiti Sains Malaysia, 16150 Kubang Kerian, Kelantan; Malaysia.

Corresponding Author: Dr. Rishya Manikam, Department of Accident and Emergency Medicine, University Malaya Medical Centre, 59100 Kuala Lumpur, Malaysia; Phone: +0060123292226; Fax: +0060379494198; Email: rishyaman@yahoo.com

Received: 16 January 2011

Accepted: 04 April 2011

Published: 31 May 2011 condition warrants a comprehensive orthopaedic management which results in a good clinical outcome. We also review various treatment modalities described before for the treatment of periprosthetic fractures.

Keywords: Floating total knee, Total knee arthroplasty, Periprosthetic fracture

$* * * * * * * * *$

Manikam R, Kumar CS, Mohamad N. Reversed tibial locking plates in management of traumatic ipsilateral periprosthetic fractures of the distal femur and proximal tibia after total knee arthroplasty. International Journal of Case Reports and Images 2011;2(5):21-25.

\section{$* * * * * * * * *$}

doi:10.5348/ijcri-2011-05-34-CR-4

\section{INTRODUCTION}

Periprosthetic fractures following total knee arthroplasty are potentially devastating complications to both the patient and the surgeon. It is reported that the complication rates of treatment for periprosthetic fractures following total knee arthroplasty range widely from $25-75 \%$ [1]. However, all the reported fractures were isolated to either femur or tibia. To date, ipsilateral periprosthetic fractures of the distal femur and proximal tibia after total knee arthroplasty are rarely encountered and it signifies a management challenge as there are only two other case reports describing the management of this condition [2]. We report an alternative approach to management of this complex fracture. 


\section{CASE REPORT}

A 50-year-old man was referred to our unit following a motor vehicle accident four days prior to his admission. He was a cyclist who was knocked down by a car from behind which injured his right knee. He did not have any other injuries. One year prior to the accident, he had undergone a right posterior stabilized total knee arthroplasty (PFC Sigma, Depuy, Warsaw, Ind) for osteoarthritis. His pre-injury range of motion of right knee was $\mathrm{O}^{\circ}$ to $120^{\circ}$. He was able to go back to work after the TKA. As he was undecided about undergoing another surgery, he initially opted only for traction. He agreed for surgery three days later. After the accident, he presented to us with complaints of pain, swelling and deformity of his right lower limb associated with inability to bear weight or ambulate. The range of motion of the right knee was grossly limited due to the pain.

Clinical examination revealed valgus deformity of his right leg, diffuse swelling, tenderness and decreased range of movements around the knee joint. His distal circulation was good and there was no alteration in the neurological examination. Plain radiographs of the knee joint revealed periprosthetic fractures with comminuted right supracondylar femur fracture and proximal third of tibia and neck of fibula fracture (Figure 1A, 1B). There was no radiographic evidence of loosening or displacements of the knee implants.

We used two eight $\mathrm{cm}$ incisions on the medial and lateral aspects of the distal thigh. Upon evacuation of the fracture haematoma, the fracture pattern was more evident. There was a six part comminuted fracture mainly affecting the supracondylar region of the femur with no extension to the implants. Most of the comminution was over the lateral part of the distal femur but the prosthesis was stable and not loosened. The reversed Proximal Lateral Tibia Locking Plates (PLT LCP, Synthes) were inserted after identifying the submuscular planes on both the medial and lateral supracondylar regions (Figure 2A, 2B). The plates were secured in position with locking screws. Even though increased resistance was encountered during drilling through the cement mantle, there was no disruption to the stability of the implants. A third incision measuring $10 \mathrm{~cm}$ was made over the lateral proximal leg. The proximal tibial fracture was a comminuted four part fracture, extending proximally to just below the tibial tray stem. The tibial tray was stable without any evidence of loosening. Single Proximal Lateral Tibia Locking Plate (PLT LCP, Synthes) was inserted after identifying the submuscular plane over the lateral aspect of the proximal tibia. Similar resistance was encountered during the drilling of the cement mantle for the placement of the locking screws but it did not jeopardise the stability of the implant. Appropriate irrigation of all the wounds was done and the wounds were closed in layers.

He made an uneventful recovery after surgery.
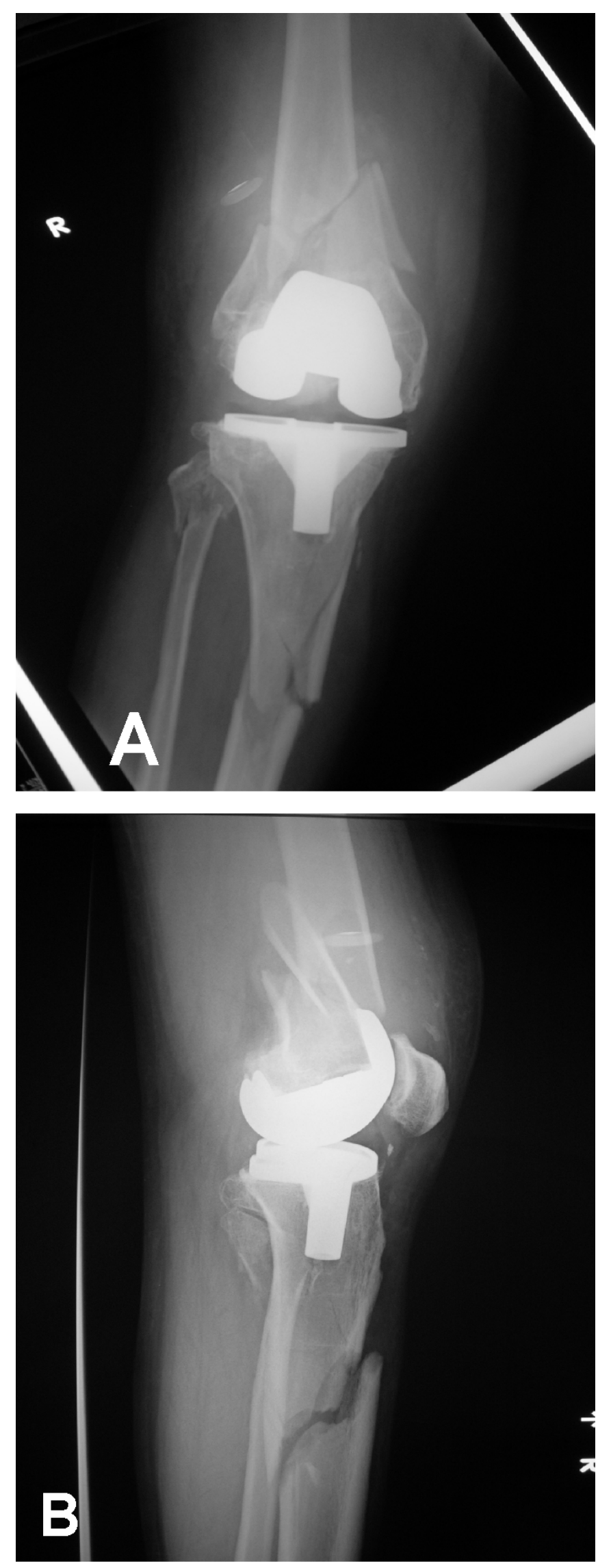

Figure 1: A) Anteroposterior view of periprosthetic fracture following TKA of right knee; B) Lateral view of periprosthetic fracture following TKA of right knee. 

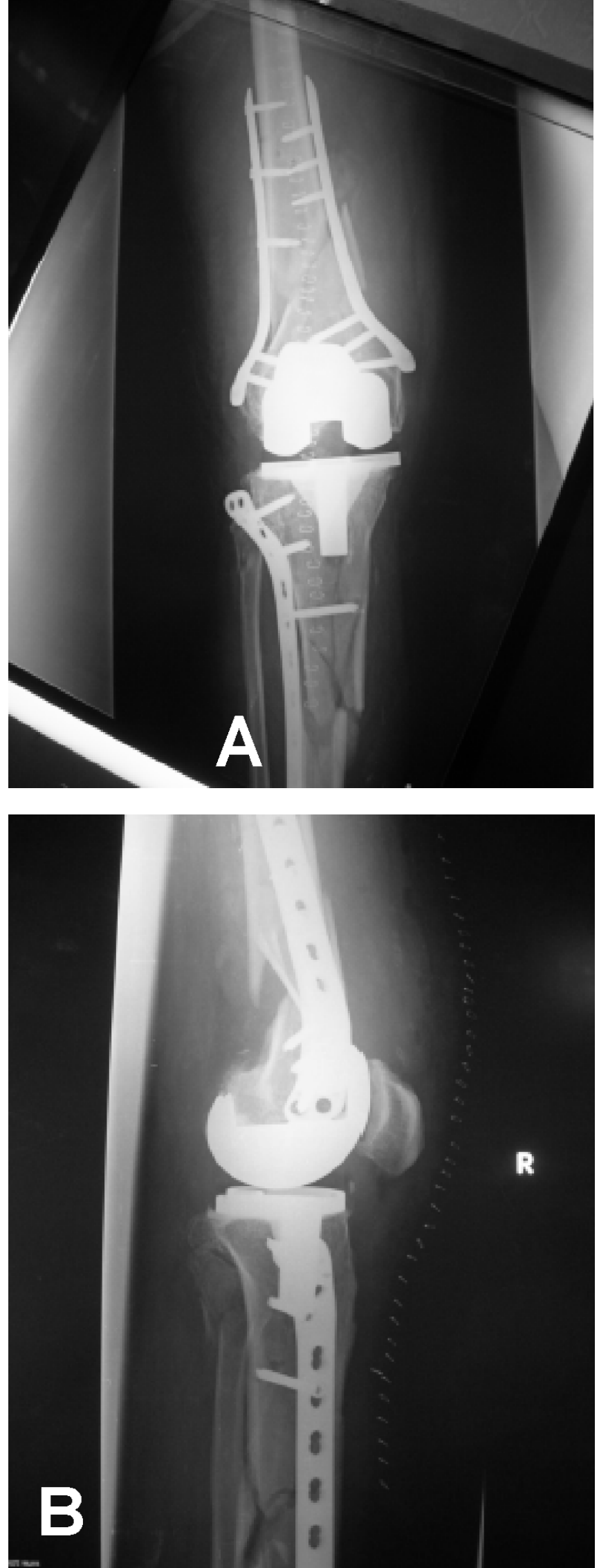

Figure 2: A) Post-operative weight bearing anteroposterior view of right knee; B) Post- operative weight bearing lateral view of right knee.
Upon discharge on day six, he was seen by a physiotherapist and instructed for non-weight bearing ambulation in a knee brace for six weeks with the aid of crutches and encouraged to do daily knee exercises. On his visit at eight weeks, all his wounds had healed with scars and there was evidence of radiological union with callous formation. His range of motion was $10^{\circ}$ to $90^{\circ}$ at eight weeks. He was advised to commence with partial weight bearing for four more weeks. At six months follow-up, he was able to bear full weight and his range of motion of the knee improved with $10^{\circ}$ to $90^{\circ}$. On the last follow-up at 18 months after surgery, he was able to walk without any walking aids and the active range of movements of his knee was $10^{\circ}-110^{\circ}$.

\section{DISCUSSION}

Periprosthetic fractures following total knee arthtoplasty are rare injuries and it is extremely rare to have ipsilateral traumatic distal femoral and proximal tibial fracture following a total knee arthroplasty $[2,3]$. The "Floating Total Knee" situation in this case is a significant injury as it is challenging and difficult to treat. The outcome of periprosthetic fracture treatments are not encouraging [1].

Periprosthetic femoral fractures without loosening of implants are conventionally treated with casting, external fixation, plating, flexible intramedullary nail and retrograde intramedullary nail. Hurson et al. used an above-knee Ilizarov external fixator [4]. Retrograde femoral nailing and Kuntcher nails were other methods used by Dodd et al. and Tanaka et al. respectively $[5,6]$. In both the cases described by them, the femoral and tibial components were fixed firmly to the bone and it provided adequate stability until fracture union. Pleva et al., in describing their experiences treating 21 patients in their centre, have mentioned use of various types of plates, including the less common clamp plates [7]. Kaab et al. have used LISS plates for stabilization in periprosthetic fractures, even in cases of poor bone quality with good functional outcome [8]. Ricci et al. reports use of Locking Condylar Plates (Synthes, Paoli, PA) combined with minimally invasive insertion technique in treating periprosthetic supracondylar femur fractures [9]. It provided satisfactory results in non-diabetic patients. For fractures with loosened implants, the options include revision arthroplasty with long stemmed femoral component, custom made component, a tumour prosthesis and an allograft implant composite. Beharrie and Nelson treated a 67-year-old woman with displaced periprosthetic fracture and loosened tibial component with long stemmed tibial component combined with circlage wiring of the fracture site [3].

Jeong et al. have reported a case involving a 80year-old man with ipsilateral periprosthetic fractures of the distal femur and proximal tibia after total knee arthroplasty [2]. The described patient was steroiddependent for rheumatoid arthritis, low dose, and the 
injury was associated with a low-energy fall. The patient had a loose femoral component but the tibial component was well-fixed to the bone. The authors opted to revise the femoral and tibial components to a modular, highly constrained TKA with fully cemented stems that bypassed the fractures. It allowed immediate weight bearing and excellent short-term results.

Jamali et al. successfully treated an 81-year-old man with ipsilateral periprosthetic fractures of the tibia and femur following a TKA [11]. In their case, the TKA implants were stable and the use of bone specific locking plates for both the supracondylar femur and proximal tibia using the minimally invasive technique was advocated. The authors highlighted that with the combination of locking plates and minimally invasive technique used, provided successful outcome with a low intraoperative blood loss, minimal incision length, low morbidity, rigid immediate fixation, complete fracture healing and retention of components.

In our case, a high velocity trauma in a 50-year-old adult with TKA resulted in a complex injury. The implants were not loosened and quality of the distal bone stock was good. We decided to use the PLT LCP plates based on an ongoing study in our centre which showed that by reversing the plate meant for proximal tibia of one side, it fitted well with distal femur of the opposite side. The unique morphology of the plate also provided good purchase of the bone. We defined the outcome as satisfactory because all of the criteria described by Chen et al. were met; these criteria include 1) a healed fracture, 2) anatomic or near anatomic alignment permitting satisfactory function and 3) patient satisfaction [10].

\section{CONCLUSION}

The "Floating Total Knee" in this case was a rare and significant injury. The outcome of the treatment was encouraging. Although we realize that the usefulness of data based on a single case is limited, we are encouraged by the outcome in this case and believe that PLT LCP is a viable option in floating total knee.

$* * * * * * * * *$

\section{Author Contributions}

Rishya Manikam - Conception of design, Acquisation of data, Analysis and interpretation of data, Drafting the article, Critical revision of the article, Final approval of the version to be published.

CS Kumar - Conception of design, Acquisation of data, Analysis and interpretation of data, Drafting the article, Critical revision of the article, Final approval of the version to be published.

Nasir Mohamad - Conception of design, Acquisation of data, Analysis and interpretation of data, Drafting the article, Critical revision of the article, Final approval of the version to be published.

\section{Guarantor}

The corresponding author is the guarantor of submission

\section{Conflict of Interest}

Authors declare no conflict of interest.

\section{Copyright}

(C) Rishya Manikam et al. 2011; This article is distributed under the terms of Creative Commons attribution 3.0 License which permits unrestricted use, distribution and reproduction in any means provided the original authors and original publisher are properly credited. (Please see www.ijcasereportsandimages.com /copyright-policy.php for more information.)

\section{REFERENCES}

1. Douglas A, Dennis MD.Periprosthetic Fractures following Total Knee Arthroplasty. J Bone. Joint Surg Am 2001;83:120-130

2. Jeong GK, Pettrone SK, Liporace FA, Meere PA."Floating total knee": ipsilateral periprosthetic fractures of the distal femur and proximal tibia after total knee arthroplasty. J Arthroplasty 2006;21(1):138-140.

3. Beharrie AW, Nelson CL.Impaction bone-grafting in the treatment of a periprosthetic fracture of the tibia: a case report. J Bone Joint Surg Am 2003;85A(4):703-707.

4. Hurson C, Synnott K, McCormack D.Above-knee Ilizarov external fixation for early periprosthetic supracondylar femoral fracture--a case report. Knee 2005;12(1):145-147.

5. Dodd MB, Ukachi-Lois J, Naique S, Bradnock B.Widening the gap: A difficulty encountered with retrograde intramedullary nail fixation of distal periprosthetic femoral fractures. Injury 2006;37(8):302-306.

6. Tanaka Y, Kobayashi T, Ohashi M, Kaneko D, Nemoto K.A new operative procedure using a Kuntcher nail for a periprosthetic supracondylar femoral fracture after revision total knee arthroplasty: A case report. Knee 2007;14(1):59-62.

7. Pleva L, Sír M, Madeja R.Our experiences with the treatment of periprosthetic fractures of femur. Biomed Pap Med Fac Univ Palacky Olomouc Czech Repub 2004;148(1):75-79.

8. Kääb MJ, Stöckle U, Schütz M, Stefansky J, Perka C, Haas NP.Stabilisation of periprosthetic fractures with angular stable internal fixation: a report of 13 cases. Arch Orthop Trauma Surg 2005;126(2):105110.

9. Ricci WM, Loftus T, Cox C, Borrelli J.Locked plates combined with minimally invasive insertion technique for the treatment of periprosthetic supracondylar femur fractures above a total knee arthroplasty. J Orthop Trauma 2006;20(3):190196. 
10. Chen F, Mont MA, Bachner RS.Management of ipsilateral supracondylar femur fractures following total knee arthroplasty. J Arthroplasty 1994;9(5):521-526.

11. Amir A. Jamali, Mark A. Lee, Rakesh Donthineni and John P. Meehan. Minimally Invasive Management of a Floating Prosthesis Injury with Locking Plates. J Arthroplasty 2007;22(6):928-933. 